\title{
Time-frequency analysis of electroencephalogram series
}

\author{
S. Blanco, ${ }^{1}$ R. Quian Quiroga, ${ }^{1}$ O. A. Rosso, ${ }^{2}$ and S. Kochen ${ }^{3}$ \\ ${ }^{1}$ Departamento de Física, Facultad de Ciencias Exactas y Naturales, Universidad de Buenos Aires, Pabellón I, \\ Ciudad Universitaria, 1428 Buenos Aires, Argentina \\ ${ }^{2}$ Instituto de Cálculo, Facultad de Ciencias Exactas y Naturales, Universidad de Buenos Aires, Pabellón II, \\ Ciudad Universitaria, 1428 Buenos Aires, Argentina \\ ${ }^{3}$ Centro Municipal de Epilepsia, Divisíon Neurología, Hospital Ramos Mejía, Universidad de Buenos Aires, Urquiza 609,
} 1221 Buenos Aires, Argentina

(Received 29 August 1994)

\begin{abstract}
In this paper we propose a method, based on the Gabor transform, to quantify and visualize the time evolution of the traditional frequency bands defined in the analysis of electroencephalogram (EEG) series. The information obtained in this way can be used for the information transfer analyses of the epileptic seizure as well as for their characterization. We found an optimal correlation between EEG visual inspection and the proposed method in the characterization of paroxism, spikes, and other transient alterations of background activity. The dynamical changes during an epileptic seizure are shown through the phase portrait. The method proposed was examplified with EEG series obtained with depth electrodes in refractory epileptic patients.
\end{abstract}

PACS number(s): 87.90. $+\mathrm{y}, 02.70 . \mathrm{Hm}$

\section{INTRODUCTION}

It has been well over a century since it was discovered that the mammalian brain generates a small but measurable electrical signal. The electroencephalogram (EEG) of small animals was measured by Caton in 1875 , and in man by Berger in 1925 . It had been thought by the mathematician Wiener, among others, that generalized harmonic analysis would provide the mathematical tools necessary to penetrate the mysterious relations between the EEG time series and the functioning of the brain. The progress along this path has been slow however, and the understanding and interpretation of EEG's remain quite elusive.

Traditional EEG tracing is now interpreted in much the same way as it was done 50 years ago. More channels are used now and much more is known about the clinical implication of the waves, but the basic EEG display and the quantification of the waves are quite similar to those of their predecessors of a half century ago.

There is no taxonomy of EEG patterns that delineates the correspondence between those patterns and brain activity. The clinical interpretation of EEG records is made by a complex process of visual pattern recognition and association on the part of the clinician, and significantly more often in the last years (with the introduction of the personal computers) through the use of the Fourier transform.

Quantitative EEG analysis as a field includes a wide variety of techniques. These are frequency analysis (spectral analysis), topographic mapping, compressed spectral arrays, significance probability mapping, and other complex analytical techniques [1-3]. A recent approach to the problem of the quantification of the EEG series has been presented by nonlinear dynamics [4-11].

The morphology and topography of sharp transients have been correlated with seizure type and therapeutic response to different medications and surgery. An essen- tial component of the traditional visual interpretation of the clinical EEG is the characterization of infrequent, morphologically variable transient events, especially those associated with the epilepsies ("spikes," "spikes and waves," etc.) [1-3]. Accordingly, a great deal of energy has been spent over the years in efforts to search automatically long recordings for these phenomena and epileptiform transient detection, but with different results [12-14]. Anyway, the most diffused quantitative method in clinical practice is the spectral analysis together with a visual assessment [1-3].

When working in the frequency domain it is useful to divide EEG activities into three different categories [15]: (i) spontaneous nonparoxysmal or background, (ii) spontaneous paroxysmal activity, and (iii) activity evoked by external sensory stimulation. Consequently, it is quite obvious that in the frequency domain representation, rhythmic components are relatively enhanced at corresponding frequencies, whereas transients (for example, epileptic spikes, isolated paroxysm, etc.) are smeared over the spectrum and, therefore, are no longer recognizable. From this, it follows that the principal field of spectral analysis is the background activity, which means the first category mentioned above, whereas in the other two categories there exist only special cases to which standard spectral analysis can be successfully applied [15].

The methods mentioned above are applied to the activity analysis in a single channel, independently of the activity in other channels. The most common methods of studying interactions between two channels are the cross-correlation and the cross-spectral analyses $[15,16]$. The average amount of mutual information and nonlinear correlation are recently developed methods [17-20]. These methods all try to determine whether two channels have a common activity and, often, whether one channel contains activity induced by the activity in the other channel. Clearly proving causality is extremely difficult, but it can sometimes be inferred by measuring time 
differences.

From another point of view, an electroencephalogram (EEG) may be considered as a time series measured on a dynamical system that represents brain activity. This subject has caught the attention of several researchers in this field, having found the important feature that the variability of the EEG signals is not noise and presents an attractor $[4-6,9]$.

The treatment of EEG series using the approach of nonlinear dynamic systems has opened new possibilities for knowledge of the brain dynamic. However, the aims are not limited to this, but also to obtaining new forms to quantify differences in the EEG series that have some kind of clinical application.

The metric approach usually employed in nonlinear dynamical analysis is based on distances and assumes the stationarity of the data sets. Distances between points in appropriate embeddings of the data are used to compute a set of metric properties. These quantities are difficult to compute, require large data sets, and degrade rapidly with additive noise [21].

Mayer-Kress and Layne [5] used the reconstruction techniques in the time series of the EEG to obtain their phase portrait. These diagrams suggest chaotic attractors with divergent trajectories and therefore the EEG series seem nonstationary. This means that the average position of a series defined over some interval changes in another. Layne, Mayer-Kress, and Holzfuss [7] conclude that the EEG series are nonstationary and present high dimensionality, in which case the concepts of attractor and fractal dimension would not be applied because these are asymptotic or stationary properties of a dynamical system. However, Babloyantz and Destexhe [8] focused their attention on the fact that this nonstationarity is strictly true for awake states but could be different for states of the sleep cycles or for patients with certain pathologies.

This problem has not been well studied and it has brought about a great variety of results exposed by different authors $[6,9]$. Due to the great extension of the EEG series that is necessary for nonlinear metric treatment (satisfying all the mathematical hypotheses) a criterion of stationarity is almost impossible to satisfy in practice. Consequently, if the time series are nonstationary, the metric algorithms must not be used.

Statistical tests of stationarity have revealed a variety of results depending on conditions, with estimates of the amount of time during which the EEG is stationary ranging from several seconds to several minutes [22-25]. However, as a practical matter, whether or not the same data segment is considered stationary depends on the problem being studied, the type of analysis being performed, and the measured (features) used to characterize the data.

The analysis of stationarity can be done by using a method based on the weak stationarity criterion, which validates the application of the algorithms later used in a rigorous way, in order to obtain reliable results [25]. For all the reasons mentioned above, the inclusion of the time evolution in the quantification of the EEG series is an open problem.
In this paper we present a method based on Gabor's old idea [26] for the simultaneous treatment in the timefrequency space of a signal. The proposed methods let us analyze the time evolution of the frequency rhythm of an EEG signal and visualize the frequency engagement during epileptic activity as well as paroxysm activities. The correlation between the obtained frequency evolution series for the different channels and bands can be used to obtain some knowledge about the interaction and consequently causality between channels and bands.

The different time behaviors identified by this method can be verified by the corresponding phase portraits obtained from the associated EEG epochs. This information can be used as a first step in the formulation of dynamical models of the epileptic seizure and its propagation.

This paper is organized in the following way. In Sec. II the experimental setup and the clinical data are presented. In Sec. III an abstract of the traditional spectral analysis is given and the new time-frequency method is introduced. As an example of the method some selected results and discussion are presented for the intracranial EEG series in Sec. IV. Finally a summary is given in Sec. V.

\section{EXPERIMENTAL SETUP AND CLINICAL DATA}

Our method was applied to the EEG recording from interseizure and seizure brain activity of a refractory epileptic patient prone to surgical treatment. The EEG intracranial registers (stereo EEG) were obtained from a male patient 21 years old, during $9 \mathrm{~h}$, with 12 depth electrodes (each electrode having 5 to 15 contacts) placed in the epileptogenic zone and the propagation brain areas. Each signal was amplified and filtered using a $1-40-\mathrm{Hz}$ bandpass filter. A four-pole Butterworth filter was used as a low-pass filter and as an antialiasing scheme. We stored the signal EEG in different samples free of artifacts. Preseizure, during seizure, and postseizure signal EEG's were chosen for the quantification. From all allowed EEG signals 120 records (EEG time series) provided by the different contacts, were selected in order to perform the clinical diagnosis by the physician team, and they are the same used in the present work. The sample rate of the EEG signal was 256 data per second and the record length for the different signals varied between 1 and $3 \mathrm{~min}$. According to the visual assessment of the EEG seizure recording, this patient presented an epileptogenic area in the hippocampus with mediate propagation to the girus cingular and the supplementary motor area on the left hemisphere.

The use of depth electrodes provides records where the noise and artifact contamination effects (usually present in the EEG series obtained with scalp electrodes) are minimized. Anyway, the applicability of the proposed method is not restricted to the use of these kinds of EEG records.

\section{TIME-FREQUENCY ANALYSIS}

Spectral decomposition of the EEG by computing the Fourier transform has been used since the very early days 
of electroencephalography. The rhythmic nature of many EEG activities leads itself naturally to this analysis. Fourier transform allows the separation of various rhythms and an estimation of their frequencies independently of each other, a difficult task to perform visually if several rhythmic activities occur simultaneously. Spectral analysis can also quantify the amount of activity in a frequency band.

The spectral analysis of EEG signals has proved to be quite useful in comparing short samples of data from patients against age-matched normative values, as well as in sleep stage analysis and quantification of drug, metabolic effects, and various disease states [12]. But important information about peak timing is lost.

The results of EEG spectral analysis are often grouped into the traditional frequency bands, i.e., $\delta$ (less than 4 $\mathrm{Hz}), \theta(4-7 \mathrm{~Hz}), \alpha(8-13 \mathrm{~Hz}), \beta(14-30 \mathrm{~Hz})$, and $\gamma$ activity (above $30 \mathrm{~Hz}$ ). There is much physiological and statistical evidence for the independence of several of these bands, but their boundaries can vary a little according to the particular experiment being considered, and they can be adjusted as required [1-3]. The most popular way of performing frequency analysis has been to apply the fast Fourier transform (FFT) algorithm directly to a short (usually 1-4 sec) segment of digitized data $[12,15]$. In the methods mentioned before, the time evolution was not taken into account or, as in the case of the compress spectra, they only provided a visual tool with a difficult interpretation of the contained information.

Our aim is to obtain a description of the EEG signals in a combined time-frequency space. For this purpose, we followed the ideas that Gabor introduced in 1946 $[26,27]$, as a basic element of the proposed method. The Gabor transform is equivalent to wavelet algorithms, with a fixed window; but in the present work we employed the Gabor transform due to the following: (a) In the analyzed frequency range both methods are equivalent using an appropriate window, (b) the Gabor transform is easily comprehensible by its analogies with the Fourier transform. This topic becomes relevant in comparison with the bibliography and in the application of the method by the physician team. Then, as a first step, we performed the Gabor transform of the EEG signal denoted by $S(t)$ as follows:

$$
\mathcal{S}_{D}\left(\omega_{0}, t_{0}\right)=\int_{-\infty}^{\infty} S(t) g_{D}^{*}\left(t-t_{0}\right) e^{i \omega_{0} t} d t
$$

We used for $g_{D}(t)$ a slide Gaussian window with width $D$, as has been suggested by Gabor. According to this algorithm, one dimensional signals are represented in a combined time-frequency space [27]. These functions are situated on a lattice in this combined space, with clearances $t_{0}$ and $\omega_{0}$ in the time and frequency axes, respectively. If we represent the associated intensity to each point in the time-frequency lattice (as for example, the graph that corresponds to the EEG signal display in Fig. 1), we obtain a tridimensional pattern as is shown in Fig. 2 or its corresponding density level diagram (Fig. 3). In these two graphs, we normalized to the maximum intensity. In Fig. 2, 50 levels have been considered.

These graphs, in particular the density level diagram with color codes, can be used as a visual tool for a qualitative description of the time evolution of the different spectral frequencies contained in the EEG recording. Again, it is difficult to extract the information contained in both kinds of graphs. In this sense, the most important advantage of this method is its systematic instrumentation and the rigorous mathematical background in comparison with the compress spectra and other similar methods [15].

As a second step, we defined the evolution of the spectral frequency content $\mathcal{B}^{(i)}$ for the band $i(i=\delta, \theta, \alpha, \beta)$ defined in the frequency interval $\left(\omega_{\min }^{(i)}, \omega_{\max }^{(i)}\right)$ as

$$
\mathcal{B}^{(i)}(\omega, t)=\mathcal{G}_{D}^{*}(\omega, t) \mathcal{G}_{D}(\omega, t) \forall \omega_{\min }^{(i)} \leq \omega \leq \omega_{\max }^{(i)} ;
$$

then, the power spectral intensity for the $i$ band as a time function will be

$$
I^{(i)}(t)=\int_{\omega_{\min }^{(i)}}^{\omega_{\max }^{(i)}} \mathcal{B}^{(i)}(\omega, t) d \omega,
$$

and consequently, the total spectral power intensity is

$$
I_{T}(t)=2 \int_{0}^{\infty} \mathscr{B}(\omega, t) d \omega,
$$

where the spectral intensity content is defined in the frequency interval $(-\infty, \infty)$. Then the power spectral intensity per band relative to the total intensity will be

$$
R^{(i)}=\left(I^{(i)} / I_{T}\right) \times 100 .
$$

For the subsequent analysis of the EEG signal, we define for the different bands the mean weight frequency

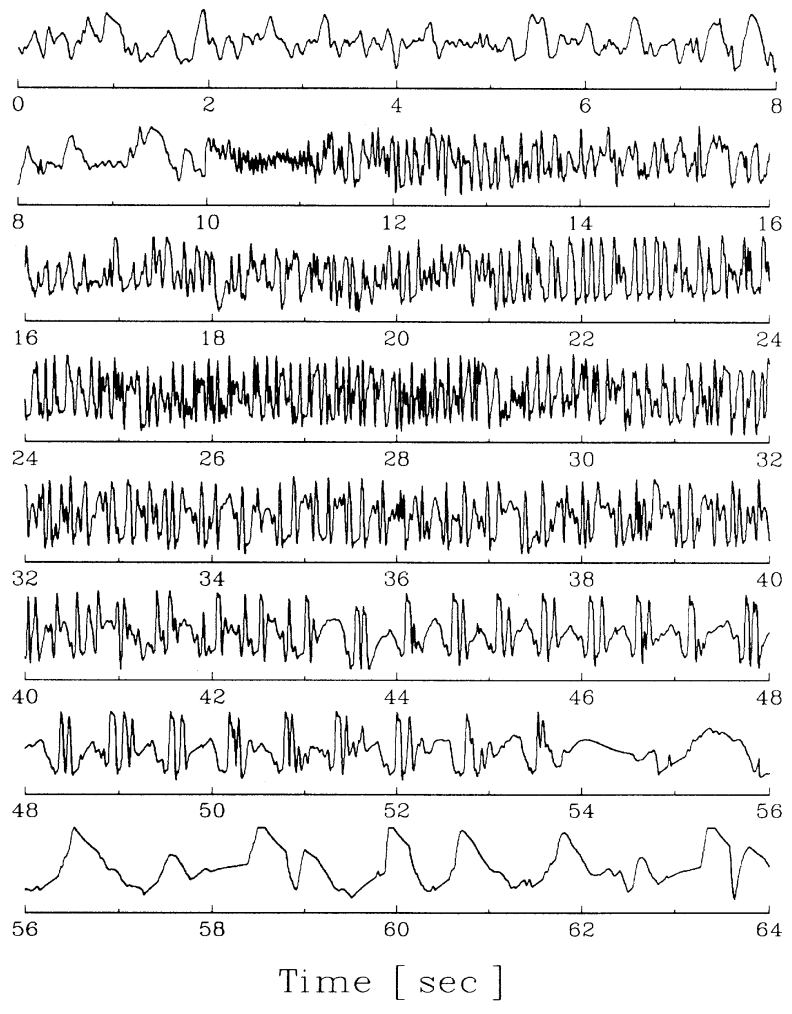

FIG. 1. Recording of the EEG signal corresponding to a depth electrode in the epiloptogenic region (left hippocampus). 


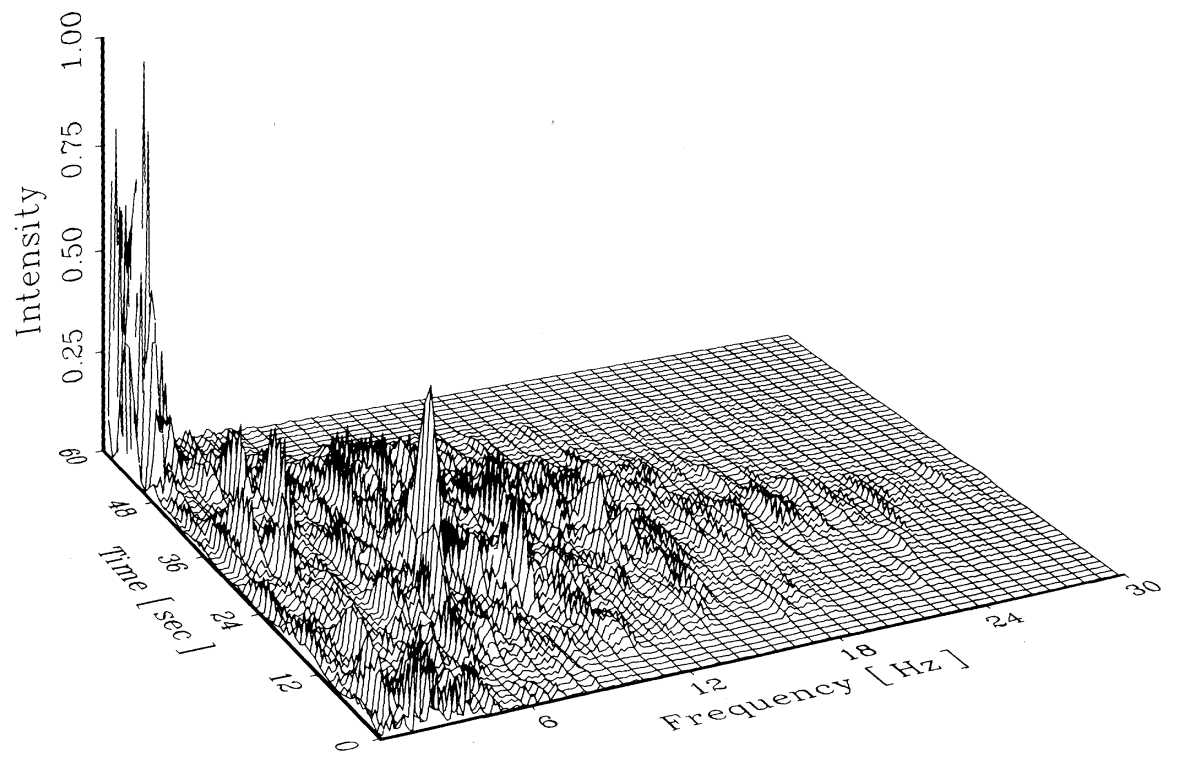

FIG. 2. Associated intensity in the time-frequency lattice for the EEG signal in Fig. 1. The dimension of the unitary shell was $\Delta \omega=0.25 \mathrm{~Hz}$ and $\Delta t=0.25$ sec.

value $\widetilde{\omega}$ at time $t$ as

$$
\widetilde{\omega}^{(i)}(t)=\left[\int_{\omega_{\min }^{(i)}}^{\omega_{\max }^{(i)}} \mathcal{B}^{(i)}(\omega, t) \omega d \omega\right) / I^{(i)}(t)
$$

and the main peak frequency in the $i$ band at time $t, \omega_{M}^{(i)}$ as the frequency value for which $\mathcal{B}^{(i)}$ takes its maximum value in the interval $\left(\omega_{\min }^{(i)}, \omega_{\max }^{(i)}\right)$. That is,

$$
\mathscr{B}^{(i)}\left(\omega_{M}, t\right)>\mathcal{B}^{(i)}(\omega, t) \forall \omega \neq \omega_{M} \in\left(\omega_{\min }^{(i)}, \omega_{\max }^{(i)}\right) .
$$

Note that when $\widetilde{\omega} \cong \omega_{M}$ we are in the presence of a quasimonofrequency signal (band). If this behavior is observed during a reasonable period we shall say that we have a frequency engagement in the corresponding frequency band.

We introduce a new parameter $\Delta^{(i)}$ and call it monofrequency deviation. This parameter, as a function of time, gives us an idea about the periods in which the engage- ments are relevant:

$$
\Delta^{(i)}(t)=\left|\widetilde{\omega}^{(i)}(t)-\omega_{M}^{(i)}(t)\right| .
$$

Moreover, in order to compare these new time series, for different bands and channels we have normalized each one to its maximum value $\left[\Delta_{N}^{(i)}(t)=\Delta^{(i)}(t) / \Delta_{\max }^{(i)}\right]$. Having introduced these new time series $R(t), \widetilde{\omega}^{(i)}(t), \omega_{M}(t)$, and $\Delta^{(i)}(t)$ is important, as they have allowed us to characterize the epileptic seizure as well as its evolution by means of quantifiable magnitudes that are independent of the signal's morphology.

\section{RESULTS AND DISCUSSION}

In order to avoid the nonstationarity problems usually present in the EEG signal we worked with a window width $D=4 \mathrm{sec}$. In this way, the stationarity hypothesis was accomplished. The sample rate of the EEG signal

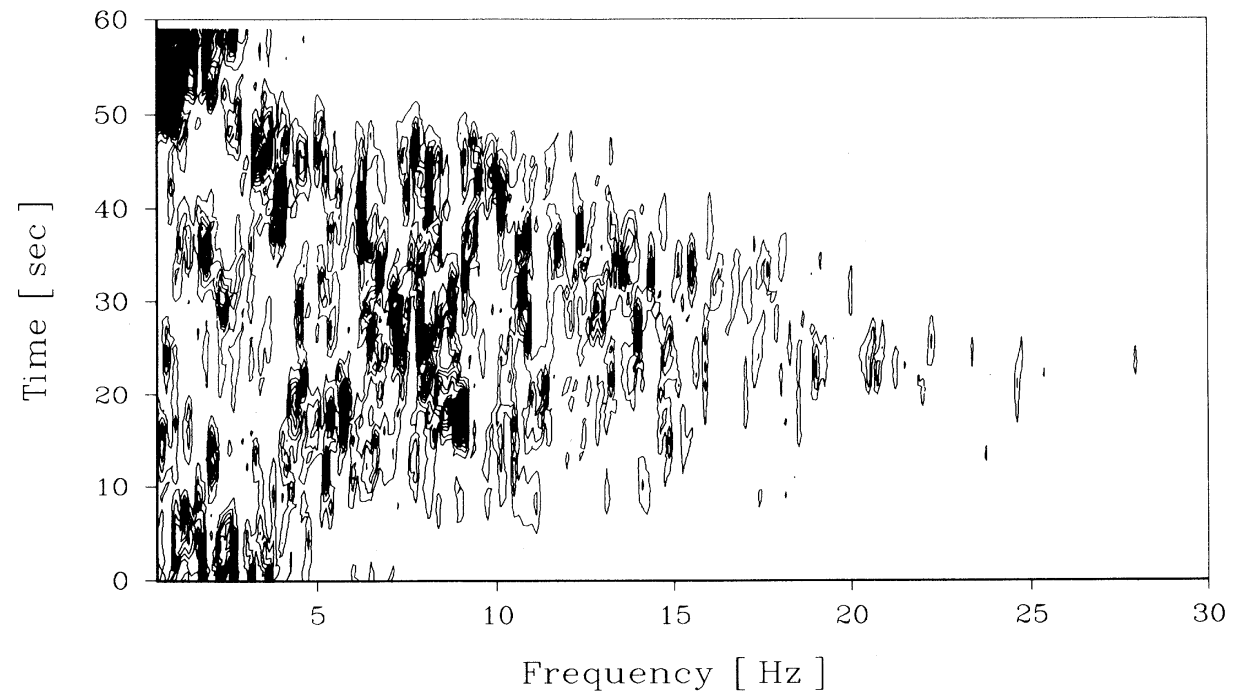

FIG. 3. Density level diagrams corresponding to the tridimensional pattern shown in Fig. 2. Fifty levels are displayed. 


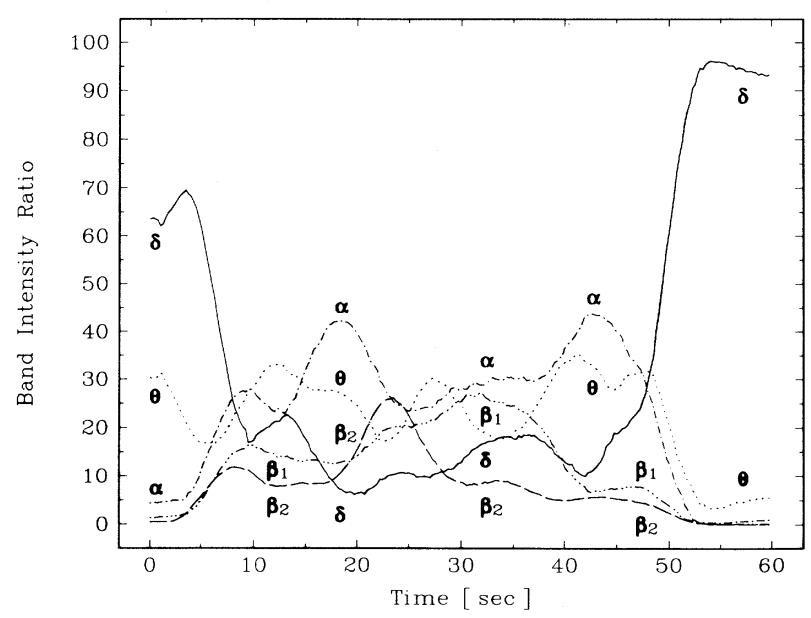

FIG. 4. Power spectral intensity per band relative to the total intensity, as a function of time for the EEG signal shown in Fig. 1.

was 256 data per second, and the displacement velocity of the slide window was 84 data. Then, the resolution in the time-frequency space was $\Delta \omega=0.125 \mathrm{~Hz}$ and $\Delta t=0.25$ sec. For the present analysis we considered the following frequency bands: $\delta(0.5-3.5 \mathrm{~Hz}) ; \theta(3.5-7.5 \mathrm{~Hz}) ; \alpha$ $(7.5-12.5 \mathrm{~Hz})$; and the $\beta$ band was divided in two, $\beta_{1}$ $(12.5-18 \mathrm{~Hz})$ and $\beta_{2}(18-30 \mathrm{~Hz})$.

In Fig. 1, the EEG signal for $64 \mathrm{sec}$ corresponding to one depth electrode in the left hippocampus region is shown. From a visual inspection, it is clear that around the 10th second the epileptic seizure starts, and finishes around the 54th second.

In Fig. 4 we display the power spectral intensity per band relative to the total intensity $R^{(i)}$ for the EEG signal shown in Fig. 1. Looking at the time evolution of these quantities, a good agreement between the changes in $R^{(i)}$ and in the signal morphology can be established. In Figs. 5-9 we show the time evolution of $\widetilde{\omega}^{(i)}$ and $\omega_{M}$ for the different bands considered in this work.

We emphasize that, when $\widetilde{\omega} \cong \omega_{M}$, a quasimonofre-

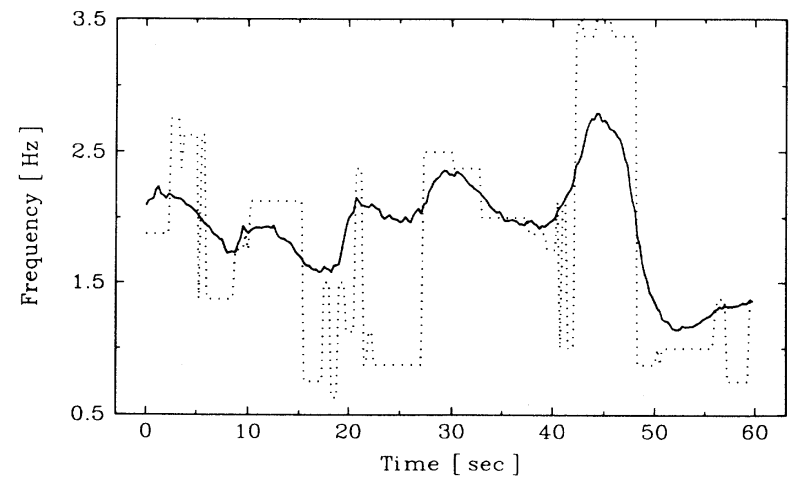

FIG. 5. Time evolution of the mean frequency $\widetilde{\omega}$ (full line) and main peak frequency $\omega_{M}$ (dotted line) for the $\delta$ band corresponding to the EEG signal of Fig. 1.

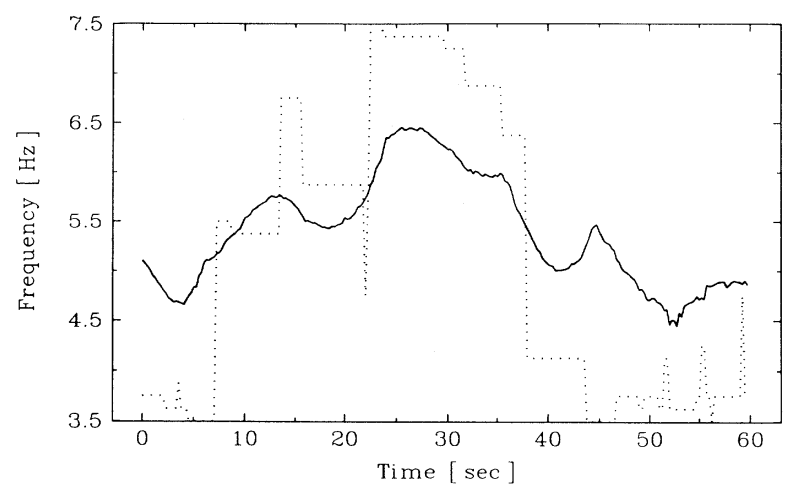

FIG. 6. Same as Fig. 5 for the $\theta$ band.

quency behavior evolves. This can be understood as a quasifrequency engagement. From the total set of signals that is allowed for analysis rises the fact that this behavior can be associated with an epileptic seizure, as well as paroxism activity.

In order to perform the analysis, we divided the signal shown in Fig. 1 into different time intervals. These intervals were suggested by the structure of Fig. 4 and are in very good agreement with the changes in the signal morphology.

$0-10 \mathrm{sec}$. Up to $5 \mathrm{sec}$ the predominance of the slow frequencies is observed, where the $\delta$ rhythm is the principal component. From $5 \mathrm{sec}$ on the fast frequencies increase and the $\alpha$ and $\theta$ rhythms are the most important near $10 \mathrm{sec}$ (Fig. 4). From Fig. 1 we can observe that around $10 \mathrm{sec}$, it is clear that the epileptic seizure starts. Between 8 and $10 \mathrm{sec}$ engagements in the $\delta\left(\Delta_{N}^{(\delta)}<5 \%\right), \theta$ $\left(\Delta_{N}^{(\theta)}<15 \%\right)$, and $\beta_{1}\left(\Delta_{N}^{\left(\beta_{1}\right)}=20 \%\right)$ bands are observed (Figs. 5, 6, and 8). Note that this engagement in the $\beta_{1}$ band is quite stable.

$10-23 \mathrm{sec}$. The predominance of the $\alpha$ rhythm is clear, with components within the $\theta$ rhythm. From the middle of this interval an increase in the intensity of the $\beta_{2}$ rhythm is observed (see Figs. 1 and 4). Clear engagements in $\delta \quad\left(\Delta_{N}^{(\delta)}<15 \%\right), \theta \quad\left(\Delta_{N}^{(\theta)} \sim 20 \%\right)$ and $\beta_{1}$ $\left(\Delta_{N}^{\left(\beta_{1}\right)} \sim 5 \%\right)$ bands, and a trend to engagement in $\alpha$ bands $\left(20 \%<\Delta_{N}^{(\alpha)}<30 \%\right)$, are observed in Figs. 5-8.

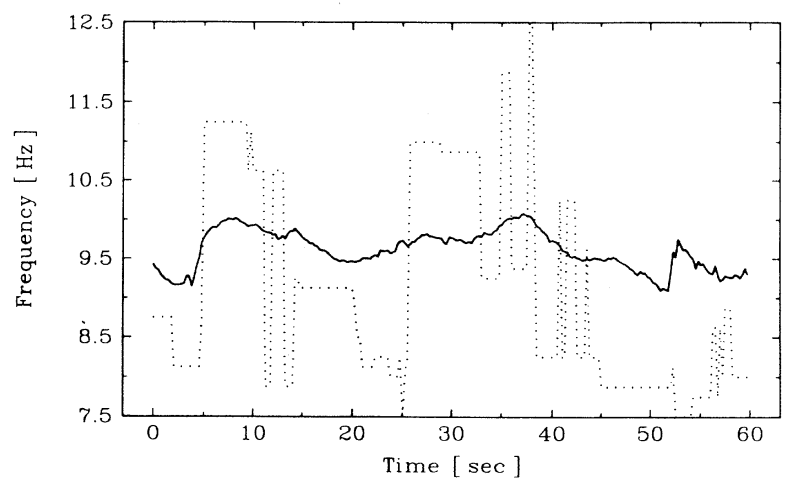

FIG. 7. Same as Fig. 5 for the $\alpha$ band. 


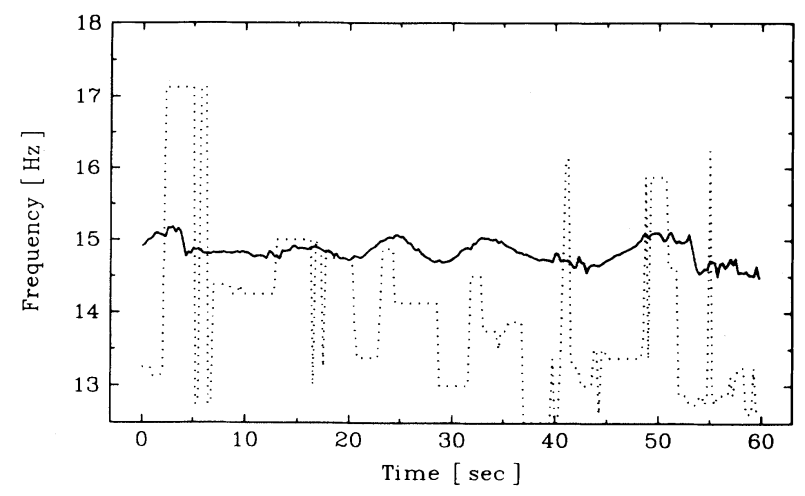

FIG. 8. Same as Fig. 5 for the $\beta_{1}$ band.

$23-38 \mathrm{sec}$. The epileptic seizure is totally developed. The frequencies in the $\alpha$ and $\beta_{1}$ bands are predominant and coexist with low frequencies in the $\theta$ band (see Figs. 1 and 4). A strong $\delta$ engagement $\left(\Delta_{N}^{(\delta)}<5 \%\right)$ can be seen (see Fig. 5).

$38-48$ sec. We can see a strong decrease in the relative intensity of the $\beta$ rhythms and an increase for the $\theta$ and $\alpha$ rhythms. The $\delta$ engagement holds in the first seconds.

$48-60 \mathrm{sec}$. The epileptic seizure is over $(54 \mathrm{sec})$. The $\delta$ rhythm is completely dominant. Only engagements in the $\delta$ band are observed at the beginning of the interval $\left(\Delta_{N}^{(\delta)} \sim 5 \%\right)$.

The previous example suggests that the epileptic seizure can be characterized during the first seconds by a strong engagement in the high frequency band $\left(\beta_{1}\right)$ and

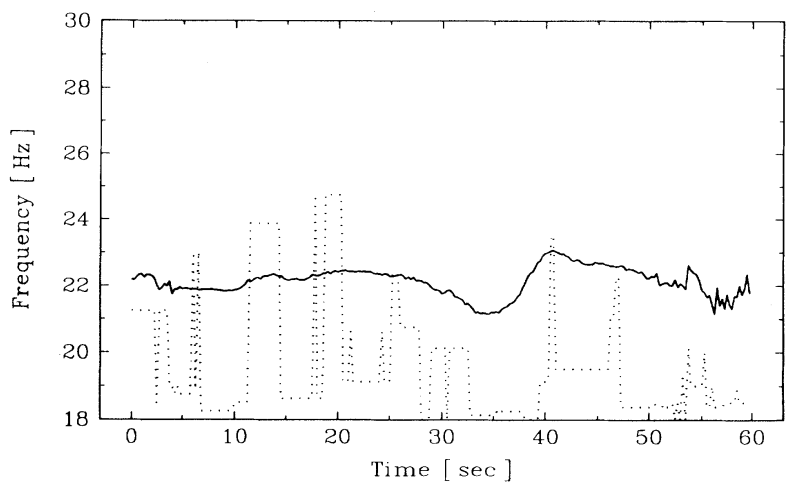

FIG. 9. Same as Fig. 5 for the $\beta_{2}$ band.

another engagement in low frequency ones. The low frequency engagement holds during the entire seizure. A similar description can be given for all the available signals of the epileptogenic and propagation zones.

As a complement, for a better comprehension of the changes in the dynamic behavior of the epileptic seizure, we show in Fig. 10 the phase portrait corresponding to the signal of Fig. 1 for the following time intervals: (a) preseizure (0-7 sec); (b) start of the seizure (14-21 sec); (c) full development of the seizure $(31-38 \mathrm{sec})$; (d) end of seizure $(45-52 \mathrm{sec})$. The time lags employed are extracted from the frequencies' engagement observed (low band engagements). We must stress from Fig. 1 that, although the seizure seems to have a predominance of high frequencies, the underlying $\delta$ frequency establishes the at-
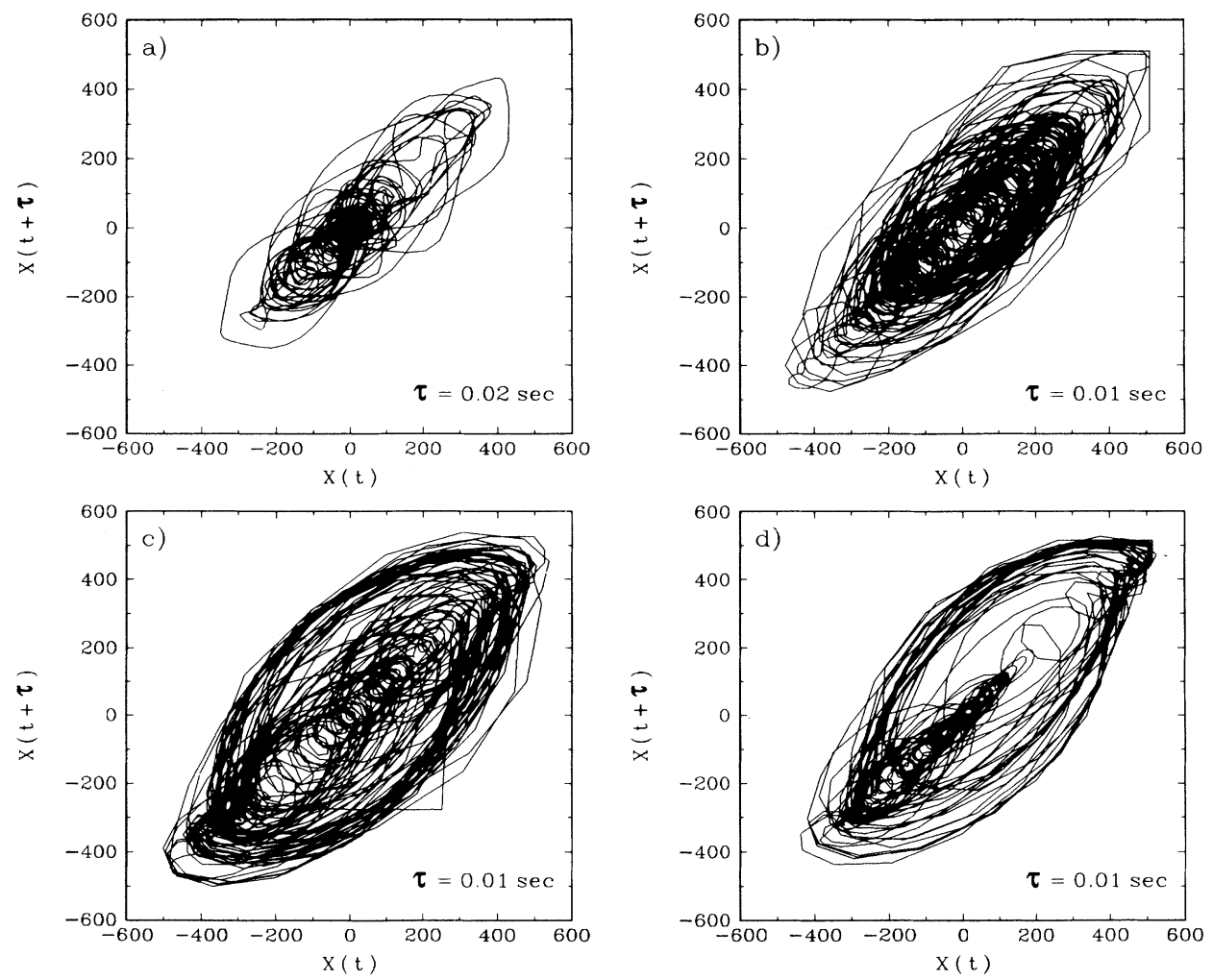

FIG. 10. Phase portrait corresponding to the signal of Fig. 1 for the time intervals (a) preseizure $(0-7 \mathrm{sec})$, (b) start of the seizure (14-21 sec), (c) full development of the seizure $(31-38 \mathrm{sec})$, and (d) end of seizure $(45-52 \mathrm{sec}) . \tau$ is the time lag employed. 


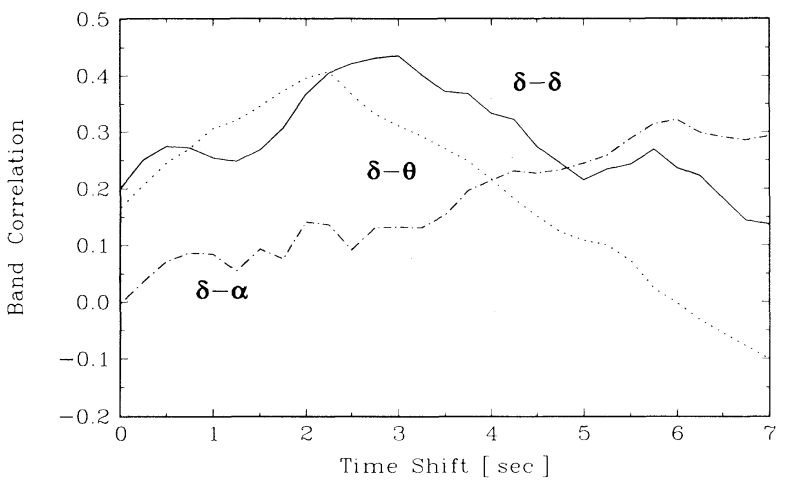

FIG. 11. Lag correlation between monofrequency deviation time series in the $\delta$ band of a channel in the epileptogenic region (Fig. 1) and the corresponding $\delta, \theta$, and $\alpha$ bands of the corresponding contralateral channel.

tractor limit cycle.

From Fig. 10 and from the previous discussion we can confirm the validity of the basic assumption that, at the seizure onset, a transition takes place in the dynamic behavior of the neuronal network from a complex behavior to a simpler one $[10,18]$. A work about a model that represents this time evolution is actually in progress.

The frequency engagements, preseizure and during seizure, suggest that a method for detecting the information transference between channels (brain zones) involved in the epileptogenic zone and the other zones of the brain can be derived. When we speak about "information transfer" we do not refer to the seizure propagation but to how the behavior in a band and in a determined channel of the epileptogenic zone affects the behavior in other bands in channels that are not so close to it.

The $\Delta^{(i)}$ parameter, as a time function, gives us an idea about the periods in which the engagements are relevant. Then we can evaluate the correlation among the time series $\Delta^{(i)}$ that arises from two different channels and the corresponding band, as a function of a time shift $\tau$. From this procedure we can investigate when an engagement in one channel and band induces similar behavior in another channel and band.

In Fig. 11 we display, as an example, the $\delta-\delta, \delta-\theta$, and $\delta$ - $\alpha$ correlations as a function of the time shift $\tau$ between a channel in the epileptogenic region and the corresponding contralateral. In Fig. 12 we show the same correlations, but now between a channel in the epileptogenic region and one in a zone outside.

It can be observed that the behaviors are very different. Figure 11 shows a high correlation in the $\delta-\delta$ and $\delta-\theta$ bands engagements. These present a maximum correlation at $\tau=3$ and $2 \mathrm{sec}$, respectively. Figure 12 shows a decreasing correlation for the two channels involved. This procedure, used for all the possible pairs of channels with their corresponding bands, can be used to identify the epileptic focus.

The behavior observed in the EEG signals analyzed in this paper lets us make the assumption that the epileptogenic zone (focus) acts as a global pacemaker with some characteristic frequency determined by the frequency engagements. From the results, we also can observe that

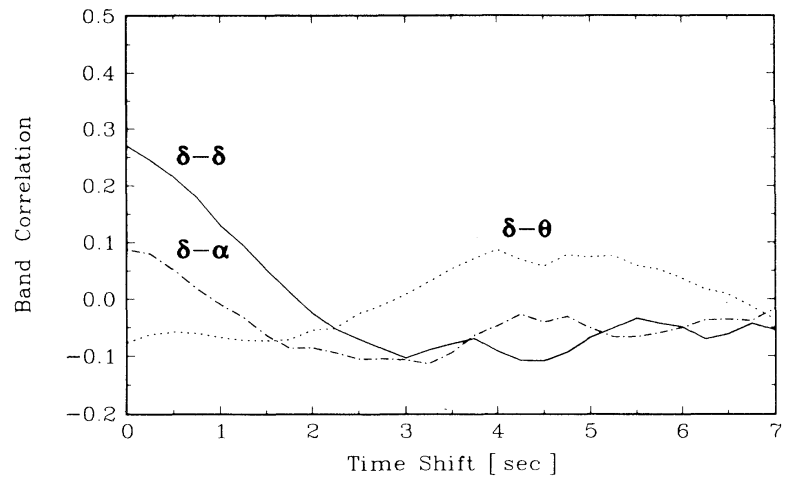

FIG. 12. Same as Fig. 11 between a channel in the epileptogenic region (Fig. 1) and one outside.

engagements in a band can induce engagements in another channel and in other bands and the corresponding time for the information transfer.

This method is a possible tool to distinguish channels in an epileptogenic region from other ones. Moreover it can be useful for obtaining valuable information in order to model the epileptic seizure.

\section{CONCLUSION}

In this paper we introduce a method that allows us to perform the EEG time-frequency analysis in a systematic way. This method lets us give an accurate description of the time evolution of the rhythm defined in the EEG characterization.

In this way we can generate time series of parameters that quantify the dynamical behavior of the brain activity in a way independent of the EEG signal morphology. In particular, the lag correlation among these time series gives good information about the process of the spread of the epileptiform activity through the brain.

We applied this method to intracranial EEG records of epileptic refractory patients. We can conclude that the epileptic seizure as well as the paroxistic activity can be characterized by a quasimono-frequency activity in some band. This characteristic can be used to detect precursors of the seizure and to study the dynamical changes in its time evolution. In particular, in this work we found good evidence that during the epileptic seizure a transition takes place in a dynamic behavior of the neuronal network from a complex to a simpler one.

The use of the present time-frequency analysis together with the clinical patient history of the visual assessment of the EEG can contribute to the identification of the source of the epileptic seizure and of its dynamic. Furthermore, it yields insights with respect to the theory of how epileptic seizures occur.

\section{ACKNOWLEDGMENTS}

This work was supported by the Consejo Nacional de Investigaciones Científicas y Tecnicas (CONICET) and Fundación Alberto J. Roemmers (Argentina). We are grateful to Dr. J. P. N. Pijn, Lic. P. Salgado, and Lic. L. Riquelme for useful discussions. 
[1] Handbook of Electroencephalography and Clinical Neurophysiology, Vol. I: Methods of Analysis of Brain Electrical and Magnetic Signals, edited by A. Gevins and A. Rémond (Elsevier, Amsterdam, 1987).

[2] Handbook of Electroencephalography and Clinical Neurophysiology, Vol. II: Clinical Applications of Computer Analysis of EEG and Other Neurophysiological Signals, edited by F. H. Lopes da Silva, Storm van Leeuwen, and A. Rémond (Elsevier, Amsterdam, 1986).

[3] Current Practice of Clinical Electroencephalography, edited by D. D. Daly and T. A. Pedley, 2nd ed. (Reven, New York, 1990).

[4] A. Babloyantz, Dimensions and Entropies in Chaotic Systems, edited by G. Meyer-Kress (Springer-Verlag, Berlin, 1986), pp. 241-245.

[5] G. Mayer-Kress and S. C. Layne, Ann. N.Y. Acad. Sci. 504, 11 (1987).

[6] Chaos in Brain Function, edited by E. Basar (SpringerVerlag, Berlin, 1990).

[7] S. C. Layne, G. Mayer-Kress, and J. Holzfuss, Dimensions and Entropies in Chaotic Systems, edited by G. MeyerKress (Springer-Verlag, Berlin, 1986), pp. 246-256.

[8] A. Babloyantz and A. Destexhe, in Proceedings of the IEEE First International Conference on Neural Networks, edited by M. Caudill and C. Butler (IEEE, San Diego, 1987), Vol. 4, pp. 31-40.

[9] J. P. N. Pijn, J. Van Neerven, A. Noest, and F. Lopes da Silva, Electroencephalogr. Clin. Neurophysiol. 79, 371 (1991).

[10] B. J. West, Fractal Physiology and Chaos in Medicine, Nonlinear Phenomena in Life Science, edited by B. J. West (World Scientific, Singapore, 1990), Vol. 1.

[11] Patterns, Information and Chaos in Neuronal Systems, Nonlinear Phenomena in Life Science, edited by B. J. West (World Scientific, Singapore, 1993), Vol. 2.

[12] A. S. Gevins, in Handbook of Electroencephalography and Clinical Neurophysiology, Vol. I: Methods of Analysis of Brain Electrical and Magnetic Signals (Ref. [1]), pp. $31-83$.

[13] F. Lopes da Silva, Electroencephalography: Basic Principles, Clinical Applications and Related Fields, edited by E.
Niedermeyer and F. Lopes da Silva (Urban and Schwarzenberg, Baltimore, MD, 1982), pp. 685-732.

[14] J. Gotman, in Handbook of Electroencephalography and Clinical Neurophysiology, Vol. II: Clinical Applications of Computer Analysis of EEG and Other Neurophysiological Signals (Ref. [2]), pp. 546-597.

[15] G. Dumermuth and L. Molinari, in Handbook of Electroencephalography and Clinical Neurophysiology, Vol. I: Methods of Analysis of Brain Electrical and Magnetic Signals (Ref. [1]), pp. 85-130.

[16] J. Gotman, in Current Practice of Clinical Electroencephalography, edited by D. D. Daly and T. A. Pedley, 2nd ed (Reven, New York, 1990), pp. 51-83.

[17] N. J. I. Mars and F. H. Lopes da Silva, in Handbook of Electroencephalography and Clinical Neurophysiology, Vol. I: Methods of Analysis of Brain Electrical and Magnetic Signals, (Ref. [1]), pp. 297-307.

[18] J. P. M. Pijn, Ph.D. thesis, Universiteit van Amsterdam, 1990.

[19] H. J. A. Beldhis, T. Suzuki, J. P. N. Pijn, P. Teisman, and F. Lopes da Silva, Eur. J. Neuroscience 5, 944 (1993).

[20] J. P. M. Pijn and F. Lopes da Silva, Basic Mechanism of the EEG, edited by S. Zschocke and E. J. Speckmann (Bishäuser, Boston, 1993).

[21] J. G. Caputo, Measures of Complexity and Chaos, Vol. 208 of NATO Advanced Study Institute Series B: Physics, edited by N. B. Abraham, A. M. Albano, A. Passamante, and P. Rapp (Plenum, New York, 1989), pp. 99-110.

[22] J. A. McEwen and G. B. Anderson, IEEE Trans. Biomed. Eng. 22, 361 (1975).

[23] T. Gasser, EEG Informatics: a Didactic Review of Methods and Applications of EEG Data Processing, edited by A. Remond (Elsevier, New York, 1977), pp. 37-55.

[24] H. Sugimoto, N. Ishii, and N. Suzumura, Comput. Programs Biomed. 7, 293 (1977).

[25] S. Blanco, H. García, R. Quian Quiroga, L. Romanelli, and O. A. Rosso, IEEE Eng. Med. Biol. Mag. (to be published).

[26] D. Gabor, J. Inst. Electron Eng. 93, 429 (1946).

[27] C. E. Heil and D. F. Walnut, SIAM (Soc. Ind. Appl. Math.) Rev. 31, 628 (1989). 